\title{
Qualidade sensorial do camarão Litopenaeus vannamei congelado
}

\section{Sensory quality of frozen shrimp Litopenaeus vannamei}

\author{
Inês Maria Barbosa Nunes Queiroga ${ }^{*}$; João Andrade da Silva²; \\ José Marcelino Oliveira Cavalheiro ${ }^{2}$; Rita de Cássia Ramos Egypto Queiroga ${ }^{3}$; \\ Ana Sancha Malveira Batista ${ }^{4}$; Tainá Amaral Barreto ${ }^{5}$
}

\section{Resumo}

O objetivo desta pesquisa foi avaliar a qualidade sensorial do camarão marinho Litopenaeus vannamei estocado sob congelamento. A análise sensorial consistiu do Método de Índice de Qualidade (MIQ) para avaliação do camarão in natura, e Análise Descritiva (AD) no camarão cozido, em amostras estocadas por um período de 90 dias, utilizando oito julgadores previamente treinados. As amostras compostas por camarão em filé (100-120 peças $/ \mathrm{kg}$ ) foram submetidas ao congelamento em Nitrogênio líquido $\left(-86^{\circ} \mathrm{C}\right)$, Túnel de Congelamento $\left(-35^{\circ} \mathrm{C}\right)$ e Freezer doméstico $\left(-18^{\circ} \mathrm{C}\right)$. Aos $0,30,60$ e 90 dias de armazenamento nesses sistemas de congelamento, foram realizadas determinações de $\mathrm{pH}$, capacidade de retenção de água (CRA), perda de peso na cocção (PPC), força de cisalhamento, cor, bases voláteis totais (BVT) e substancias reativas ao ácido tiobarbitúrico (TBARS). Os atributos de firmeza manual e maciez apresentaram melhores resultados nos congelamentos rápidos. Os parâmetros de cor $\left(\mathrm{a}^{*}, \mathrm{~b}^{*}\right.$ e L*) assim como a CRA e PPC foram maiores aos 90 dias de armazenamento, não havendo perdas significativas. Destacou-se a força de cisalhamento maior nas amostras congeladas lentamente aos 90 dias, corroborando com os dados reportados pela equipe sensorial. Aos 90 dias de armazenamento, o camarão congelado apresentou qualidade sensorial, física e química satisfatória. O tempo de prateleira deste camarão pode ser definido em cerca de 90 dias.

Palavras-chave: Maciez, cor, velocidade de congelamento, força de cisalhamento, aparência superficial

\begin{abstract}
The objective of this research was to evaluate the sensory quality of the marine shrimp Litopenaeus vannamei grown and stored in a freezer. A sensory analysis consisted of the Quality Index Method (MIQ) to review the raw shrimp and Descriptive Analysis (AD) in cooked shrimp in samples stored for a period of 90 days, using eight previously trained panelists. Accommodation comprising shrimp filet (100-120 pieces / kg) samples were subjected to freezing in liquid nitrogen $\left(-86^{\circ} \mathrm{C}\right)$, Freezing Tunnel $\left(-35^{\circ} \mathrm{C}\right)$ and Domestic Freezer $\left(-18^{\circ} \mathrm{C}\right)$. At 0, 30, 60 and 90 days of storage in these freezing systems, determination of $\mathrm{pH}$, water holding capacity (WHC), weight loss during cooking (WLC), shearing force, color, total volatile bases (TVB) and thiobarbituric acid reactive substances (TBARS) were performed. The attributes manual firmness and softness showed better results in rapid freezing.
\end{abstract}

\footnotetext{
${ }^{1}$ Discente de Pós-Graduação em Ciência e Tecnologia de Alimentos, Dept ${ }^{\circ}$ de Engenharia de Alimentos, Centro de Tecnologia, Universidade Federal da Paraíba, UFBB, Campus I, João Pessoa, PB. E-mail: inesnunes2503@hotmail.com

${ }^{2}$ Profs. Associados do Dept $^{\circ}$ de Engenharia de Alimentos, Centro de Tecnologia e Desenvolvimento Regional, UFPB, João Pessoa, PB. E-mail: joaoctdr@gmail.com; jmarcelin@uol.com.br

${ }^{3}$ Prof $^{a}$ Associada, Dept ${ }^{\circ}$ de Nutrição, Centro de Ciências da Saúde, UFPB, João Pessoa, PB. E-mail: rcqueiroga@uol.com.br

${ }^{4}$ Prof $^{\mathrm{a}}$ Adjunta, Dept ${ }^{\mathrm{o}}$ de Zootecnia, da Universidade Estadual Vale do Acaraú, UEVA, Sobral, CE. E-mail: anasancha@yahoo. com.br

${ }^{5}$ Discente de Graduação em Engenharia de Alimentos, Dept ${ }^{\circ}$ de Engenharia de Alimentos, UFPB, Campus I, João Pessoa, PB. E-mail: tainaamaral_alimentos@hotmail.com

* Autor para correspondência
} 
The color parameters $\left(\mathrm{a} *, \mathrm{~b} *\right.$ and $\left.\mathrm{L}^{*}\right)$, WHC and WCL were higher at 90 days of storage, no significant losses were observed. Highlighted the strength of greater shear in slowly frozen samples at 90 days, confirming the results reported by the sensory panel. At 90 days of storage, the frozen shrimp showed good sensory quality and physical and chemistry characteristics. The shelf life of this shrimp could be set at about 90 days.

Key words: Softness, color, speed of freezing, shearing force, surface appearance

\section{Introdução}

Uma das principais preocupações da indústria pesqueira está relacionada às tecnologias de conservação, buscando a manutenção da qualidade do produto final. Entre os vários métodos utilizados atualmente, os mais importantes são os que utilizam baixas temperaturas, capazes de preservar as características físicas, químicas e sensoriais do pescado (GONÇALVES; GINDRI JUNIOR, 2008). Camarão é o crustáceo mais importante comercializado a nível mundial (OETTERER; REGITANO; SPOTO, 2006) e congelado, é um produto de alto valor comercial e sua demanda vem aumentando, devido ao seu preço competitivo e vida útil prolongada. A temperatura de armazenamento determina, entre outros fatores relevantes, as taxas de perda de tempo de prateleira e qualidade final no momento do consumo ou utilização. As mais importantes mudanças de qualidade que ocorrem durante o armazenamento de camarão congelado são desbotamento da cor, oxidação de lipídeos, desnaturação da proteína, sublimação e recristalização do gelo que podem resultar em sabores desagradáveis, ranço, desidratação, perda de peso, perda de suculência, perda por gotejamento e alterações texturais (YAMAGATA; LOW, 1995), aumento do teor de bases voláteis e capacidade reduzida de água de ligação, bem como a deterioração microbiana e autólise. A mais alta qualidade de camarão pode ser obtida ao ser congelado imediatamente após a captura sendo importante evitar flutuações de temperatura durante o transporte e armazenamento, assim como o descongelamento e recongelamento (BOONSUMREJ et al., 2007). O objetivo desta pesquisa foi investigar o efeito de diferentes métodos de congelamento do camarão Litopenaeus vannamei armazenado durante 90 dias, sobre o tempo de prateleira e características de qualidade sensoriais.

\section{Materiais e Métodos}

\section{Matéria-prima}

Para a realização do experimento foram utilizadas amostras de camarão da espécie Litopenaeus vannamei, em filé 100-120 peças/ $\mathrm{kg}$, com 20 peças por pacote, empregando-se embalagens Cryovac seladas. As amostras foram adquiridas de uma empresa produtora, localizada no Estado da Paraíba, Brasil, em seguida transportadas em caixas isotérmicas ao LDPP (Laboratório de Desenvolvimento de Produtos Pesqueiro), DEA - CT - UFPB, para processamento e posterior realização da pesquisa. As amostras foram divididas em três partes iguais, e submetidas aos tratamentos: camarão congelado em freezer doméstico (CFD) a $-18^{\circ} \mathrm{C}$; camarão congelado em nitrogênio líquido (CNL) a $-86{ }^{\circ} \mathrm{C}$ e camarão congelado em túnel de congelamento (CTC) a $-35^{\circ} \mathrm{C}$, permanecendo armazenadas à $-18 \pm 1{ }^{\circ} \mathrm{C}$, durante o período de 90 dias para a avaliação dos parâmetros de qualidade. As determinações realizadas foram avaliadas em intervalos de 30 dias. As amostras foram descongeladas sob refrigeração $\left(4^{\circ} \mathrm{C}\right)$, por um período de 24 horas, previamente aos dias de análise.

\section{Análises físicas e químicas}

As análises químicas realizadas foram: substâncias reativas ao ácido tiobarbitúrico (TBARS) metodologia proposta por Torres e Okani (1997), e para bases voláteis totais (BVT), o 
método descrito pelo Instituto Adolfo Lutz (2008). Como análises físicas foram avaliados o potencial hidrogeniônico $(\mathrm{pH})$ pelo método no 947.05 da AOAC (2000); cor e força de cisalhamento. A cor foi determinada de acordo com a metodologia descrita por Santos, Azeredo e Martins (2008) com colorímetro digital Minolta (CR-300, Minolta, Mahwah/New Jersey, USA), para leitura dos parâmetros $L^{*}$ (luminosidade), $a^{*}$ (componente vermelho/verde) e $b^{*}$ (componente amarelo/azul). Foi empregado como condições, iluminante D65, ângulo de visão $8^{\circ}$, ângulo padrão do observador $10^{\circ}$ e especular incluída, conforme especificações da Comission Internationale de L'éclairage (CIE, 1986). A força de cisalhamento foi avaliada em texturômetro utilizando célula de carga de $25 \mathrm{~kg}$ e o programa Texture Expert for Windows, versão 1.19. $O$ teste foi realizado com uma velocidade de $2 \mathrm{~mm} / \mathrm{s}$ e com uma distância de $22 \mathrm{~mm}$. As amostras foram cortadas em cilindros de $20 \mathrm{~mm}$ de comprimento e empregou-se probe Warner-Bratzler (HDP/WBV). Foram ainda avaliados: capacidade de retenção de água (CRA) de acordo com a metodologia descrita por Hoffmam et al. (1982) e Rendimento na Cocção conforme o método proposto por Seabra et al. (2002). Todas as análises foram realizadas em triplicada.

\section{Análises sensoriais}

Recrutamento, pré-seleção dos julgadores e treinamento

Para o recrutamento empregou-se um questionário distribuído para 20 consumidores do produto, considerando as condições de saúde (fumante ou não/ tomando alguma medição/ alérgico a algum componente natural ou farmacêutico), disponibilidade horária, habilidade para descrição das percepções sensoriais (atributos) e emprego de escalas de intensidade, que assinaram um termo de consentimento livre e esclarecido, conforme exigido pela Resolução n. 196 de 10/10/1996 do Conselho Nacional de Saúde. (DELLA MODESTA,
1994; STONE; SIEDEL, 1998; BRASIL, 2003). O projeto foi aprovado pelo Comitê de Ética do Centro de Ciências e Saúde da UFPB, sob registro 09780712.8.0000.5188, e parecer de número 190.504.

Os julgadores foram submetidos ao teste dos gostos básicos (duas sessões); teste para avaliar a habilidade dos julgadores em mensurar proporcionalidade de escalas não estruturadas (duas sessões), testes triangulares e de dureza com amostras representativas (recém-capturadas/ frescas e armazenadas sob diferentes temperaturas e períodos de armazenamento), para avaliar a habilidade dos provadores em diferenciar sensorialmente as amostras (ABNT, 1993). Este processo foi dividido em oito sessões.

Oito julgadores selecionados considerando-se como critério o acerto de pelo menos $70 \%$ dos testes com uso de escalas, $90 \%$ nos testes com os gostos básicos e $70 \%$ de acerto nos testes triangulares.

$\mathrm{O}$ treinamento deu-se a seguir: realizado em nove sessões divididas em três dias, em intervalos de 3 horas entre cada sessão, utilizando exemplares de camarão para cada tempo de armazenamento, de acordo com os tratamentos utilizados. As amostras permaneceram embaladas em um recipiente com água e gelo a $4{ }^{\circ} \mathrm{C}$, de onde eram retiradas 30 minutos antes de cada sessão, e apresentadas aos julgadores, primeiro in natura (MIQ), depois cozidas(Análise Descritiva), para que os mesmos discutissem os atributos sensoriais de aparência, odor e firmeza manual (camarão in natura), e, cor, textura, aroma e sabor (camarão cozido) nos diferentes tratamentos e diferentes tempos de estocagem.

\section{Desenvolvimento do $M I Q$}

A metodologia utilizada para o Método de Índice de Qualidade foi previamente baseada em pesquisas realizadas por Martinsdottir et al. (2002) e Sveinsdottir et al. (2003). Baseia-se em parâmetros sensoriais significantes para o pescado cru em 
um sistema de escore de 0 a 2 pontos de demérito (JONSDOTTIR, 1992). Adicionalmente uma lista de atributos sensoriais que melhor descreviam as diferenças entre as amostras foi feita de forma consensual entre os julgadores pré-treinados com o auxílio de referencias (Tabela 1). A análise foi feita em três sessões por dia com dois tratamentos em cada sessão, com intervalo mínimo de 3 horas entre as sessões, no período de armazenamento $(0,30,60$, e 90 dias), resultando em 48 sessões no total.

Tabela 1. Método de Índice de Qualidade desenvolvido para camarão marinho Litopenaeus vannnamei em filé congelado.

\begin{tabular}{|c|c|c|c|}
\hline Parâmetros & & Características & Escore \\
\hline \multirow{12}{*}{ Aspecto geral } & \multirow{3}{*}{ Odor } & Característico, fresco, algas marinhas & 0 \\
\hline & & Neutro & 1 \\
\hline & & Ligeiramente acre ou rançoso & 2 \\
\hline & \multirow{3}{*}{$\begin{array}{l}\text { Aparência } \\
\text { Superficial }\end{array}$} & Brilhante, translúcida e branca & 0 \\
\hline & & Brilho menos intenso & 1 \\
\hline & & Carne opaca, esbranquiçada ou leitosa & 2 \\
\hline & \multirow{3}{*}{ Cor } & Cinza sem pontos escuros bem definidos & 0 \\
\hline & & Cinza amarelado com pontos escuros & 1 \\
\hline & & Alaranjado a vermelho & \\
\hline & \multirow{3}{*}{ Firmeza manual } & Muito rígida e firme & 0 \\
\hline & & Ligeiramente mole & 1 \\
\hline & & Flácidas/borrachudas & 2 \\
\hline Índice de qualidade (esc & & & $(0-8)$ \\
\hline
\end{tabular}

Fonte: Adaptada de Oliveira (2005).

As amostras in natura foram apresentadas em bandejas de fundo branco, devidamente codificadas, com números aleatórios de três dígitos.

\section{Análise descritiva}

Foi realizada baseando-se nos procedimentos descritos por Stone e Sidel (1998). Os termos descritos abordaram os atributos principais de cor, textura, sabor e aroma das amostras nos diferentes tratamentos utilizados. No Tabela 2 encontram- se as definições dos atributos avaliados durante as sessões. A avaliação foi feita com quatro tratamentos por sessão, com duas sessões por dia de armazenamento. Os resultados são correspondentes a três repetições por julgador.

As amostras foram envoltas em papel alumínio e cozidas durante 5 min. em água em ebulição, servidas em copos plásticos de $50 \mathrm{ml}$ a $35^{\circ} \mathrm{C}$, devidamente codificados com números aleatórios de três dígitos (NIRMAL; BENJAKUL, 2011). 
Tabela 2. Definição dos atributos sensoriais utilizados pelos julgadores que avaliaram as amostras de camarão cozido.

\begin{tabular}{ll}
\hline Atributos & Definição \\
\hline $\begin{array}{l}\text { Cor vermelha } \\
\begin{array}{l}\text { Aroma característico de } \\
\text { camarão fresco }\end{array}\end{array}$ & $\begin{array}{l}\text { Coloração da carne cozida variando de coral vivo a mais esbranquiçada com brilho } \\
\text { e umidade aparentes. }\end{array}$ \\
$\begin{array}{l}\text { Sabor característico de } \\
\text { camarão fresco }\end{array}$ & Sabor de crustáceos marinhos, algas marinhas, suave. \\
Textura & $\begin{array}{l}\text { Maciez = Força necessária para ruptura da carne na primeira mordida. } \\
\text { Suculência = Lubrificação da amostra durante a mastigação, liberação de água pelo } \\
\text { produto durante a mastigação. }\end{array}$ \\
\hline
\end{tabular}

Fonte: Oliveira (2005).

\section{Análise estatística}

A análise estatística dos dados físicos, químicos de frescor e sensoriais foi realizada por meio de análises de variância (ANOVA), analisados em função do tempo de armazenamento, e, para a comparação das médias, as mesmas foram submetidas ao teste de Tukey ao nível de $5 \%$ de significância e regressão linear, utilizando o programa SISVAR, versão 5.3 (FERREIRA, 2008).

\section{Resultados e Discussão}

Efeito do congelamento sobre as análises físicas e químicas

Não houve diferença $(p>0,05)$ entre os tratamentos em relação ao $\mathrm{pH}$ (Tabela 3 ), porém os valores aumentaram em função do tempo de maneira homogênea. Tsironi et al. (2009) ao avaliarem a vida de prateleira do camarão congelado em diferentes temperaturas, relataram $\mathrm{pH}$ inicial de 6,95 aumentando para 7,93 e 7,85 após 39 dias (-12 $\left.{ }^{\circ} \mathrm{C}\right)$ e $74 \operatorname{dias}\left(-15^{\circ} \mathrm{C}\right)$.

Nos parâmetros a* e b* (Tabela 3), não houve diferença entre os tratamentos $(p>0,05)$, havendo um aumento no final do período de armazenamento. Bak et al. (1999) reportaram para o camarão Pandalus borealis congelado, decréscimo da cor vermelha durante estocagem por 12 meses em amostras em contato com o oxigênio. Benjakul et al. (2008) relatam que o valor de a* varia de acordo com o teor de carotenóide de cada espécie, alimentação e localização geográfica. Bono et al. (2012), reportaram valores que variaram de 13,6 a 21,6 para o parâmetro $b^{*}$ no camarão vermelho, Aristaeomorpha foliácea.

$\mathrm{O}$ parâmetro $L^{*}$ (Tabela 3) não variou (p $>0,05$ ) entre os, porém aumentou ao longo do tempo mostrando valores mais altos aos 90 dias de estocagem. Desta forma o camarão analisado, pode ser considerado de elevada luminosidade, tendendo a cores mais claras com tempo de armazenamento. Cadun, Kisla e Cakli (2008), ao avaliarem camarão Parapenaeus longirostris congelado, encontraram valores variando de 48,9 para 72,9 no parâmetro L* após 75 dias de armazenamento.

Os valores de CRA e PPC (Tabela 3) não apresentaram diferença $(p>0,05)$ entre os tratamentos analisados, entretanto aumentaram no ultimo dia de armazenamento. Quando os alimentos tem elevada capacidade de reter água, a perda de peso durante o armazenamento é reduzida. Esses resultados apresentam-se superiores aos obtidos por Rocha e Rocha (2010), para camarão da mesma espécie. 
Tabela 3. Caracterização física e química, em função do tempo em cada tratamento para Litopenaeus vannamei congelado.

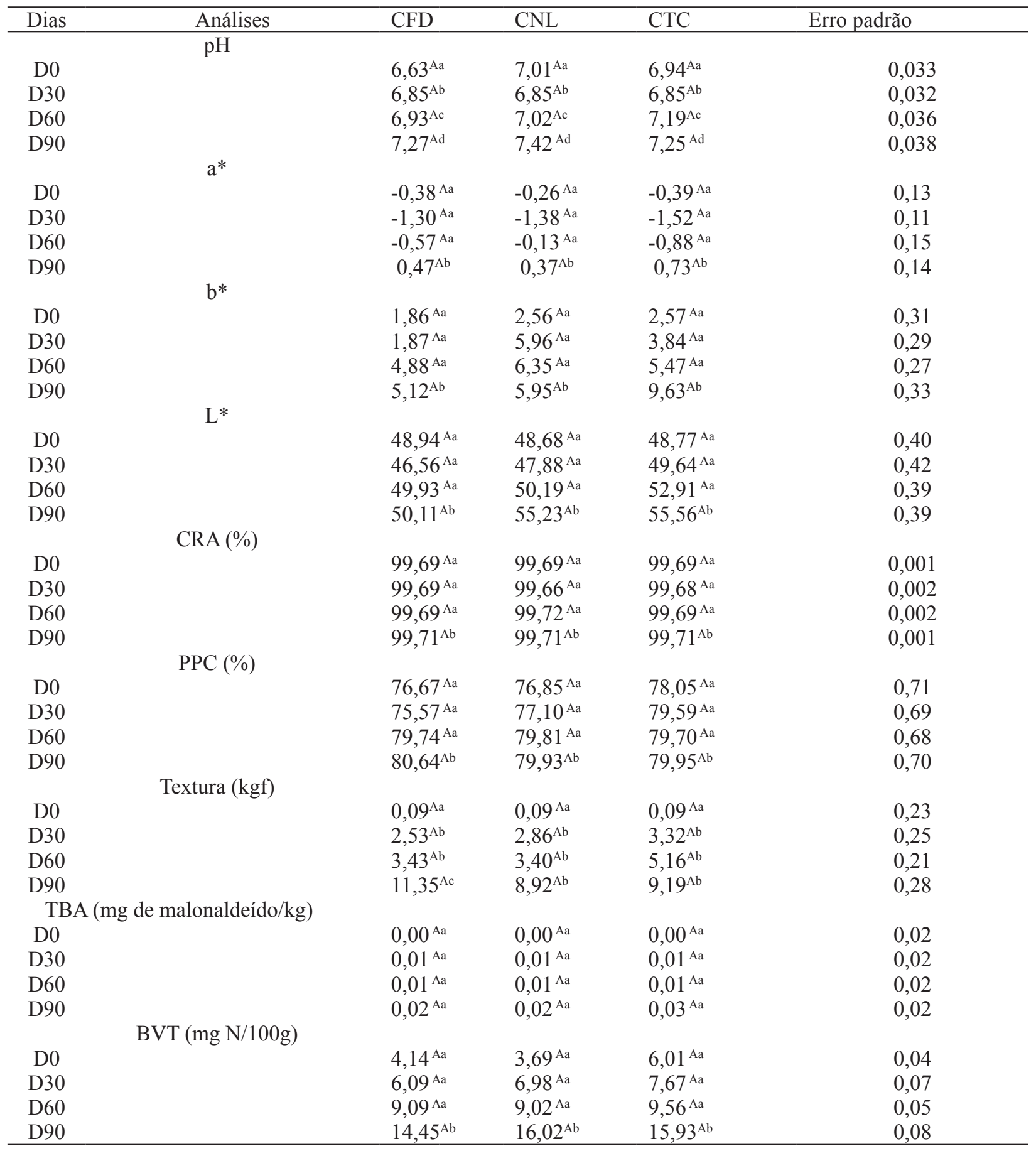

Letras maiúsculas distintas na linha indicam diferença entre tratamentos pelo teste de Tukey a 5\%., Letras minúsculas distintas na coluna indicam diferença entre tempos pelo teste de Tukey a 5\%.. CFC - congelado em freezer doméstico; CNL - congelado no nitrogênio líquido; CTC - congelado em túnel de congelamento, análises realizadas em triplicatas genuínas.

Fonte: Elaboração dos autores. 
Em relação a textura (Tabela 3) houve diferença $(p<0,05)$ entre os métodos de congelamento rápido e o lento. Os valores aumentaram em função do tempo, com destaque para as amostras submetidas ao congelamento lento que apresentaram valores mais elevados aos 90 dias. A carne descongelada tende a apresentar valores maiores de textura do que a carne in natura (HALE, WATERS, 1981). Boonsumerj et al. (2007) detectaram um aumento da força de cisalhamento, em pesquisa sobre os efeitos do congelamento e descongelamento nas variações da qualidade do camarão tigre (Penaeus monodon) congelado. O congelamentodescongelamento provoca alterações nas células musculares, resultando na liberação de enzimas a partir de mitocôndrias no sarcoplasma (HAMM, 1979).

Os valores de TBA (Tabela 3) não apresentaram diferença $(p<0,05)$ em relação aos tratamentos em função do tempo de estocagem, demonstrando a manutenção na qualidade química das amostras, com inibição da oxidação lipídica. Bak et al. (1999) analisando o camarão Pandalus borealis encontraram níveis de até $8,32 \mathrm{mg}$ de malonaldeído/ $\mathrm{kg}$ no produto armazenado por até três meses. Já Tsirone et al. (2009) encontraram valores de 0,22 e $0,25 \mathrm{mg}$ malonaldeído $/ \mathrm{kg}$ em camarão congelado a $-12{ }^{\circ} \mathrm{C} \mathrm{e}-15^{\circ} \mathrm{C}$, respectivamente. As amostras deste estudo podem ser consideradas hábeis, apresentando valores abaixo de 3,0 $\mathrm{mg}$ de malonaldeído/kg de amostra, sendo os limites de oxidação lipídica para o consumo de $7-8 \mathrm{mg}$ de malonaldeído/kg no alimento (CADUN; KISLA; CAKLI, 2008).

Nos valores de BVT não houve diferença entre os tratamentos $(p>0,05)$, em função do tempo os valores aumentaram aos 90 dias. Bono et al. (2012) avaliaram os níveis de BVT durante a estocagem a $-18^{\circ} \mathrm{C}$ e observaram resultados de até $86 \mathrm{mgN} / 100 \mathrm{~g}$ após oito meses de estocagem. Tsirone et al. (2009) encontraram valores de até $25 \mathrm{mgN} / 100 \mathrm{~g}$ após oito meses em camarão armazenado $\mathrm{a}-15{ }^{\circ} \mathrm{C}$.

\section{Método de índice de qualidade}

O esquema do MIQ desenvolvido para as amostras obteve uma soma total de pontos no valor oito e a soma total desses pontos (escores) é designada como o Índice de Qualidade (IQ).

$\mathrm{O}$ odor das amostras mostrou-se neutro à ligeiramente rançoso aos 90 dias de armazenamento (Tabela 1). Quanto a firmeza manual, as amostras submetidas ao congelamento em freezer doméstico (lento) mostraram-se ligeiramente moles aos 30 dias, à flácidas e borrachudas aos 90 dias. Houve manutenção da firmeza nos demais métodos de congelamento (rápidos), com amostras ligeiramente moles aos 90 dias, confirmando que os resultados obtidos na textura física foram percebidos pelo painel. Yamagata e Low (1995) relataram mudanças na textura de camarão congelado de firme a macia após sete semanas a $-10^{\circ} \mathrm{C}$, e em camarão estocado a $-20{ }^{\circ} \mathrm{C}$ a textura mostrou-se levemente flácida após 6 meses. Gonçalves e Gindri Junior (2008) encontraram resultados semelhantes para filés de peixe, camarão e mexilhão, e concluíram que a retenção de umidade e a alta capacidade de retenção de água no produto pode proporcionar benefícios em termos de textura. Desta maneira os resultados desta pesquisa podem corroborar com os mencionados em Fernandez, Aspe e Roecke (2009), que elegeram os atributos de odor e textura como indicadores de rejeição em filés de salmão.

A aparência superficial e a cor apresentaram amostras de brilho menos intenso e coloração cinza amarelada aos 90 dias, de forma similar ao já descrito pela avaliação instrumental da cor (Tabela 3) obtendo-se assim amostras de coloração e aparência de aceitabilidade satisfatória até o fim do período de estocagem. Diaz-Tenório et al. (2007) encontraram elevados níveis de escurecimento em camarão branco congelado e descongelado após 30 dias de armazenamento. Benjakul e Nirmal (2010) avaliando o efeito da temperatura de descongelamento no camarão Litopenaeus vannamei observou escurecimento enzimático 
após oito dias de armazenamento sob refrigeração. Tsironi et al. (2009), observaram que após 11 meses de estocagem, corroborando com os resultados desta pesquisa, o camarão acondicionado a $-12{ }^{\circ} \mathrm{C}$ e $15^{\circ} \mathrm{C}$ teve aparência aceitável.

Pode ser observado na Figura 1 que os valores de IQ, referentes aos atributos de qualidade, aumentam com o tempo de estocagem em todos os tratamentos e sugerem que as amostras tratadas com os congelamentos rápidos (amostras b e c) perderam qualidade mais lentamente que as amostras do congelamento lento (a), como pode ser visualizado pelo coeficiente angular das regressões lineares mostradas. Martinsdóttir, Sveinsdóttir e Hyldig (2002), Bonilla, Sveinsdottir e Martinsdottir (2007) e Marinho (2011), em pesquisas realizadas para o desenvolvimento de MIQ em espécies de pescado, verificaram que as médias dos escores se tornaram menos variáveis entre si à medida que o tempo de estocagem aumentava, provavelmente porque as mudanças se tornam mais evidentes com o tempo de estocagem. Entretanto, apesar das pontuações do presente estudo seguirem uma mesma tendência de concordância com os referidos autores, houve uma mudança maior aos 30 e 60 dias de avaliação, podendo ser considerado como o período intermediário entre o aceitável e o início das modificações indesejáveis nas características. A partir dos escores obtidos na avaliação sensorial, das modificações ocorridas com o tempo de estocagem e de dados microbiológicos (ANJOS, 2012) sugerese o índice de qualidade para este camarão seja de 4,0, equivalentes ao tempo de prateleira de 90 dias de estocagem sob congelamento. Resultados semelhantes foram relatados na literatura por VazPires, Seixas e Barbosa (2004), Vaz-Pires (2006), Bonilla, Sveinsdottir e Martinsdottir (2007), Teixeira et al. (2009) e Sant'Ana, Soares e VazPires (2011).

Figura 1. Escores médios do Índice de Qualidade (IQ) do camarão Litopenaeus vannamei congelado nos tratamentos analisados em função do tempo. (a. CFC - congelado em freezer doméstico; b. CNL - congelado no nitrogênio líquido; c. CTC - congelado em túnel de congelamento).
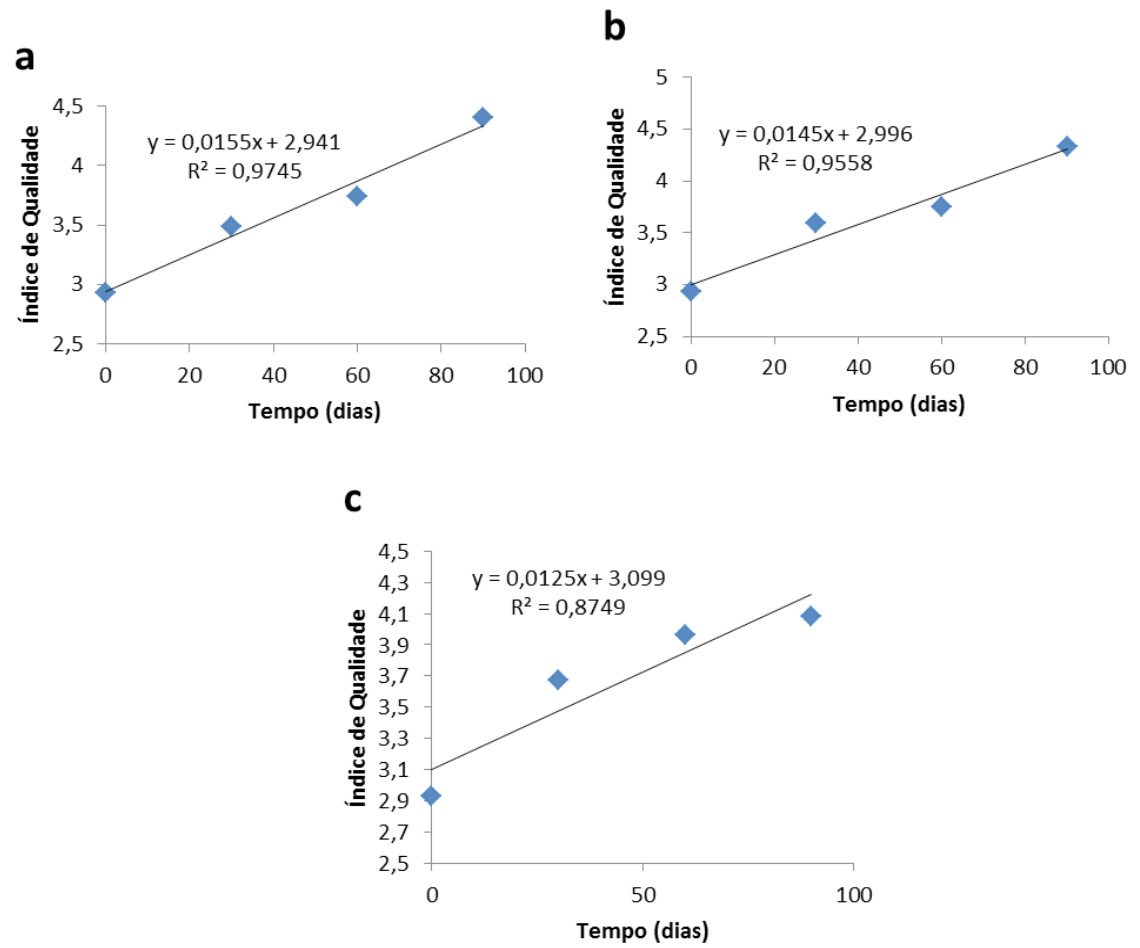

Fonte: Elaboração do autores. 


\section{Análise descritiva}

Não houve diferença $(\mathrm{p}>0,05)$ entre os tratamentos em relação à cor vermelha, os valores diminuíram gradualmente em função do tempo, considerando-se a amostra de cor vermelha clara aos 90 dias (Tabela 4). Segundo Erickson et al. (2007) a mudança mais notável em camarão branco congelado é a intensidade na coloração vermelho em sua superfície, provavelmente devida a desnaturação da parte proteica da astaxantina (pigmento natural mais abundante) que é azulado ou verde no seu estado conjugado.
Para o sabor e aroma não houve diferença entre os tratamentos $(p>0,05)$, entretanto em função do tempo os valores foram reduzidos, indicando amostras de sabor e aroma fracos, no fim do período de estocagem (Tabela 4). Pons-Sánchez et al. (2006) encontraram resultados semelhantes em anchovas do Mediterrâneo Engraulis encrasicholus. Erickson et al. (2007) encontraram valores que demonstraram sabor e aroma mais desejável em amostras congeladas de camarão branco quando comparadas a amostras frescas, porém de caráter suave.

Tabela 4. Avaliação Sensorial Descritiva realizada em função do tempo em cada tratamento para Litopenaeus vannamei cozido.

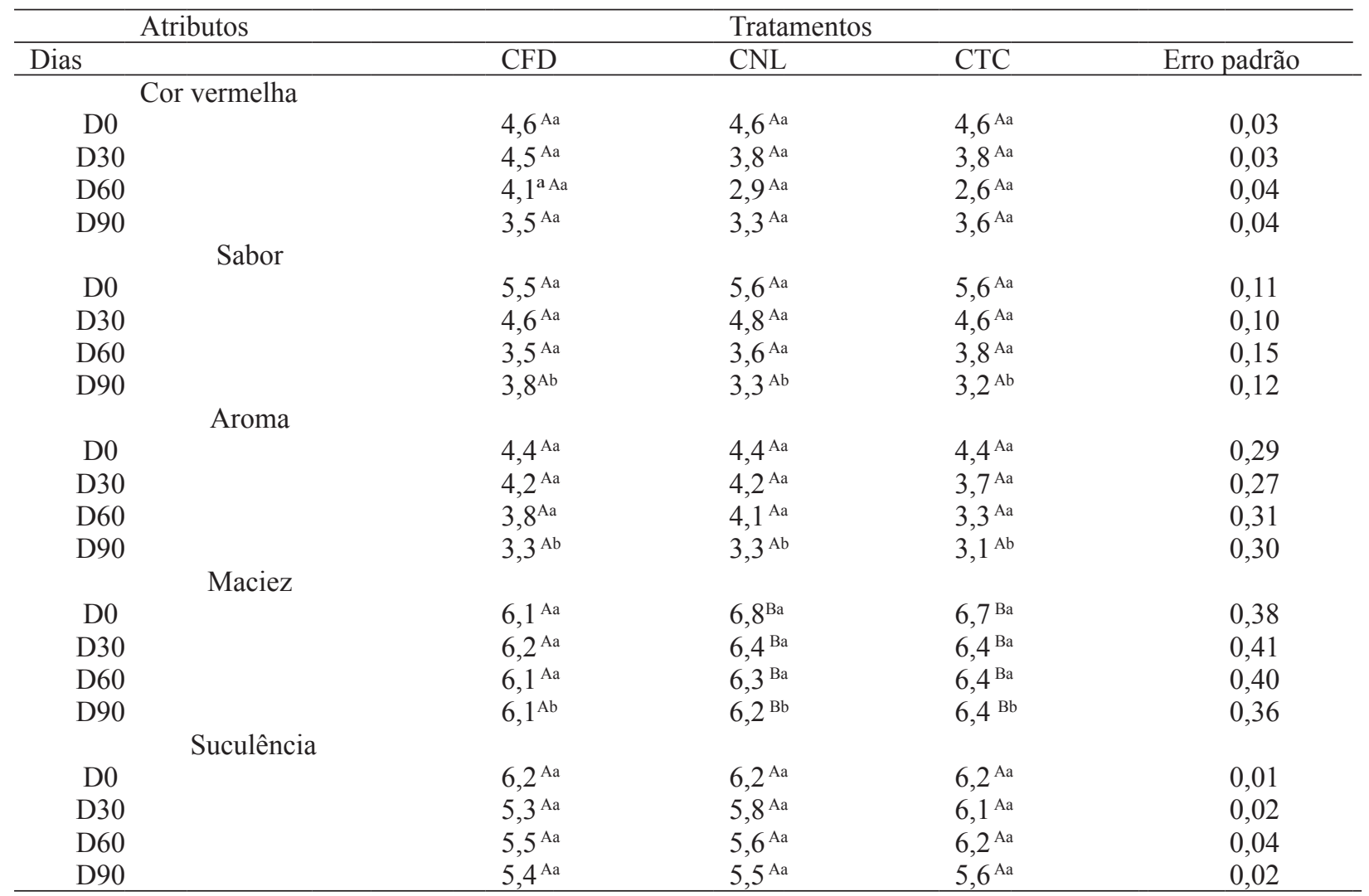

Letras maiúsculas distintas na linha indicam diferença entre tratamentos pelo teste de Tukey a 5\%., Letras minúsculas distintas na coluna indicam diferença entre tempos pelo teste de Tukey a 5\%.. CFC - congelado em freezer doméstico; CNL - congelado no nitrogênio líquido; CTC - congelado em túnel de congelamento.

Fonte: Elaboração dos autores. 
No atributo de maciez, houve diferença entre os tratamentos $(p<0,05)$ entre os tratamentos, o congelamento lento mostrou redução na intensidade desse atributo com amostras consideradas de baixa maciez aos 90 dias (Tabela 4). Já a suculência não diferiu ( $p>0,05)$ entre os tratamentos e foi mantida durante o período de armazenamento. Estes resultados corroboram com Erickson et al. (2007) que analisando camarão branco, fresco e congelado, averiguou perda na firmeza e manutenção na suculência nas amostras congeladas, sendo as amostras frescas consideradas mais suculentas e de menor firmeza.

\section{Conclusões}

Os métodos de congelamento rápido (túnel e nitrogênio líquido) apresentaram relevante eficácia na manutenção da qualidade em relação ao método de congelamento lento (freezer), com destaque para o atributo de firmeza manual e maciez que apresentaram melhores resultados nos congelamentos rápidos. Com o aumento do tempo de armazenamento foram observadas alterações na cor e na força de cisalhamento, tanto nos resultados instrumentais como na avaliação sensorial e um aumento no $\mathrm{pH}$ acompanhado de sensível elevação das BVT. A qualidade sensorial do camarão marinho Litopenaeus vannamei foi considerada satisfatória ao longo dos 90 dias de armazenamento.

\section{Agradecimentos}

À FINEP, através da RECARCINA (Rede de carnicicultura nacional) pelo financiamento da pesquisa e ao CNPq pela bolsa concedida.

\section{Referências}

ASSOCIAÇÃO BRASILEIRA DE NORMAS TÉCNICAS. NBR 12806/93: definições das etapas básicas dos fluxos de operações em estabelecimentos produtores/fornecedores de alimentos. Rio de Janeiro, 1993.
ANJOS, A. G. Emprego do frio na conservação do camarão cultivado (Litopenaeus vannamei). 2012. Dissertação (Mestrado em Ciência e Tecnologia de Alimentos) - Universidade Federal da Paraíba, João Pessoa.

ASSOCIATION OF OFFICIAL ANALYTICAL CHEMISTS - AOAC. Official methods of analysis. Washington D.C.: AOAC, 2000. 1018 p.

BAK, L. S.; ANDERSEN, A. B.; ANDERSEN, E. M.; BERTELSEN, G. Efect of modified atmosphere packaging on oxidative changes in frozen stored cold water shrimp (Pandalus borealis). Food Chemistry, Barking, v. 64, n. 2, p.169-175, 1999.

BENJAKUL, S.; VISESSANGUAN, W.; KIJROONGROJANA, K.; SRIKET, P. Effect of heating on physical properties and microstructure of black tiger shrimp (Penaeus monodon) and white shrimp (Penaeus vannamei) meats. International Journal of Food Science and Technology, Malden, v. 43, n. 6, p. 1066-1072, 2008.

BENJAKUL, S.; NIRMAL, N. P. Effect of catechin and ferulic acid on melanosis and quality of Pacific white shrimp subjected to freeze-thawing prior refrigerated storage. Food Control, Vurrey, v. 21, n. 9, p. 1263-1271, 2010 .

BONILLA, A. C.; SVEINSDOTTIR, K.; MARTINSDOTTIR, E. Development of quality index (QIM) scheme for fresh cod (Gadus morhua) fillets and application in shelf life study. Food Control, Vurrey, v. 18, n. 4, p. 352-358, 2007.

BONO, G.; GAI, F.; PEIRETTI, P. G.; BADALUCCO, C.; BRUGIAPAGLIA, A.; SIRAGUSA, G.; PALMEGIANO, G. B. Chemical and nutritional characterisation of the Central Mediterranean Giant red shrimp (Aristaeomorpha foliacea): Influence of trophic and geographical factors on flesh quality. Food Chemistry, Barking, v. 130, n. 1, p. 104-110, 2012.

BOONSUMREJ, S.; CHAIWANICHSIRI, S.; TANTRATIAN, S.; SUZUKI, T.; TAKAI, R. Effects of freezing and thawing on the quality changes of tiger shrimp (Penaeus monodon) frozen by air-blast and cryogenic freezing. Journal of Food Engineering, London, v. 80, n. 1, p. 292-299, 2007.

BRASIL. Ministério de Agricultura, Pecuária e Abastecimento. Instrução Normativa n. 62, de 26 de agosto de 2003. Métodos analíticos oficiais para análises microbiológicas para controle de produtos de origem animal e água. Diário Oficial [da] União, Brasília, DF, 18 set. 2003. Seção 1, p. 14. 
CADUN, A.; KISLA, D.; CAKLI, S. Marination of deep-water pink shrimp with rosemary extract and the determination of its shelf-life. Food Chemistry, Barking, v. 109, n. 1, p. 81-87, 2008.

COMISSION INTERNATIONALE DE L'ÉCLAIRAGE - CIE. Technical report. Viena: CIE, v. 15, n. 2, 1986.

DELLA MODESTA, R. C. Manual de análise sensorial de alimentos e bebidas: prática. Rio de Janeiro: EMBRAPA CTAA, 1994. 67 p.

DIAZ-TENORIO, L. M.; GARCIA-CARRENO, F. L.; PACHECO-AGUILAR, R. Comparison of freezing and thawing treatments on muscle properties of whiteleg shrimp (Litopenaeus vannamei). Journal of Food Biochemistry, Malden, v. 31, n. 5, p. 563-576, 2007.

ERICKSON, M. C.; BULGARELLI, M. A.; RESURRECCION, A. V. A.; VENDETTI, R. A.; GATES, K. A. Sensory differentiation of shrimp using a trained descriptive analysis panel. $L W T-$ Food Science and Technology, London, v.40, n. 10, p. 1774-1783, 2007.

FERNANDEZ, K.; ASPE, E.; ROECKE, M. L. Shelflife extension on fillets of Atlantic Salmon (Salmo salar) using natural additives, superchilling and modified atmosphere packaging. Food Control, Vurrey, v. 20, n. 11, p. 1036-1042, 2009.

FERREIRA, D. F. SISVAR: um programa para análises e ensino de estatística. Revista Symposium, Lavras, v. 6, n. 2, p. 36-41, 2008.

GONÇALVES, A. A.; GINDRI JUNIOR, C. S. G. Optimization of the freezing process of red shrimp (Pleoticus muelleri) previously treated with phosphates, International Journal of Refrigeration, Vurrey, v. 31, n. 7, p. 1134-1144, 2008.

HALE, M. B.; WATERS, M. E. Frozen storage stability of whole and headless reshwater prawns Machrobranchium rosenbergii. Marine Fish Review, Seatle, v. 42, p. 18-21, 1981.

HAMM, R. Delocalization of mitochondrial enzymes during freezing and thawing of skeletal muscle. In FENNEMA, O. R. (Ed.). Proteins at low temperatures. Washington, DC: American Chemical Society, Advances in Chemistry Series, 1979. v. 180, p. 191-654.

INSTITUTO ADOLFO LUTZ - IAL. Normas analíticas do Instituto Adolfo Lutz. 4. ed. São Paulo, v. 1, 2008. $1018 \mathrm{p}$.

JONSDOTTIR S. Quality index method and TQM system. In: OLAFSSON, R.; INGTHORSSON, A. H. (Ed.). Quality issues in the fish industry. Reykjavik: The Research Liaison Office, University of Iceland, 1992. p. 81-94.
MARINHO, L. S. Critérios para avaliação da qualidade da piramutaba (brachyplatystoma vaillantii) inteira estocada em gelo. 2011. Tese (Doutorado em Higiene Veterinária e Processamento Tecnológico de Produtos de Origem Animal) - Universidade Federal Fluminense, Belém.

MARTINSDÓTTIR, G.; SVEINSDÓTTIR, K.; HYLDIG, B. Application of quality index method (QIM) scheme in shelf-life study of farmed Atlantic Salmon (Salmo salar). Journal of Food Science, Chicago, v. 67, n. 4, p. 1570-1579, 2002.

NIRMAL, N. P.; BENJAKUL, S. Effect of ferulic acid on inhibition of polyphenoloxidase and quality changes of Pacific white shrimp (Litopenaeus vannamei) during iced storage. Food Chemistry, Barking, v. 116, n. 1, p. 323-331, 2011.

OETTERER, M.; REGITANO, M. A. B. R.; SPOTO, M. Proteínas do pescado-processamento com intervenção protéica. In: OETTERER, M.; REGITANO D'ARCE, M. A.; SPOTO, M. H. F. Fundamentos de ciência e tecnologia de alimentos. Barueri: Manole, 2006. p. 99134.

OLIVEIRA, V. M. Estudo da qualidade do camarão branco do pacífico (Litopenaeus vannamei). 2005. Tese (Doutorado em Higiene Veterinária e Processamento Tecnológico de POA) - Faculdade de Veterinária. Universidade Federal Fluminense, Niterói.

PONS-SÁNCHEZ-CASCADO, S.; VIDAL-CAROU, M. C.; NUNES, M. L.; VECIANA-NOGUÉS, M. T. Sensory analysis to assess the freshness of Mediterranean anchovies (Engraulis encrasicholus) stored in ice. Food Control, Vurrey, v. 17, n. 7, p. 564-569, 2006.

ROCHA, I. P.; ROCHA, D. M. Análise da produção e do mercado interno e externo do camarão cultivado. Revista da $A B C C$, Natal, v. 1, n. 1, p. 18-23, 2010.

SANT'ANA, L. S.; SOARES, S.; VAZ-PIRES, P. Development of a quality index method (QIM) sensory and study of shelf life of ice-stored blackspot seabream (Pagellus bogaraveo). LWT - Food Science and Technology, London, v. 44, n. 10, p. 2253-2259, 2011.

SANTOS, F. L.; AZEREDO, V. B.; MARTINS, A S. A. Efeito do fornecimento de ração complementada com semente de linhaça sobre os macronutrientes e colesterol em tecidos de camarões da Malásia (Macrobrachium rosenbergii). Ciência e Tecnologia de Alimentos, Campinas, v. 27, n. 4, p. 851-855, 2008. 
SEABRA, L. M. J.; ZAPATA, J. F. F.; NOGUEIRA, C. M.; DANTAS, M. A.; ALMEIDA, R. B. Fécula de mandioca e farinha de aveia como substituinte de gordura na formulação de hambúrguer de carne ovina. Ciência e Tecnologia de Alimentos, Campinas, v. 22, n. 3, p. 245248, 2002.

STONE, L.; SIDEL, J. L. Quantitative descriptive analyses: developments, applications, and the future. Food Technology, Chicago, v. 52, n. 8, p. 48-52, 1998.

SVEINSDOTTIR, K.;HYLDIG, G.; MARTINSDOTTIR, E.; JORGENSEN, B.; KRISTBERGSSON, K. Quality index method (QIM) scheme developed for farmed Atlantic salmon (Salmo salar). Food Quality and Preference, Barking, v. 14, n. 3, p. 237-245, 2003.

TEIXEIRA, M. S.; BORGES, A.; FRANCO, R. M.; SÃO CLEMENTE, S. C.; FREITAS, M. Q. Método de índice de qualidade (QIM): desenvolvimento de um protocolo sensorial para corvina (Micropogonias furnieri). Revista Brasileira de Ciência Veterinária, Rio de Janeiro, v. 16, n. 2, p. 83-88, 2009.
TSIRONI, T.; DERMESONLOUOGLOUA, E.; GIANNAKOUROUA, M.; TAOUKIS, P. Shelf life modelling of frozen shrimp at variable temperature conditions. Food Science and Technology, London, v. 42, n. 2, p. 664-671, 2009.

VAZ-PIRES, P.; SEIXAS, P.; BARBOSA, A. Aquaculture potential of the common octopus (Octopus vulgaris Cuvier, 1797): a review. Aquaculture, London, v. 238, n. 1-4, p. 221-238, 2004.

VAZ-PIRES, P.; SEIXAS, P. Development of new quality index method (QIM) schemes for cuttlefish (Sepia officinalis) and broadtail shortfin squid (Illex coindetii). Food Control, Vurrey, v. 17, p. 942-949, 2006.

YAMAGATA, M.; LOW, L. K. Banana shrimp, Penaeus merguiensis, quality changes during iced and frozen storage. Journal of Food Science, Chicago, v. 60, n. 4, p. 721-726, 1995. 